\title{
CAPACIDAD DE CARGA DE MUROS DE FÁBRICA SIMPLE SEGÚN DIFERENTES NORMAS. DESARROLLO DE UN MODELO ESTRUCTURAL SIMPLIFICADO $\left(^{\star}\right)$
}

\author{
(CAPACITY OF LOAD BEARING MASONRY WALLS ACCORDING TO \\ DIFFERENT STANDARDS. DEVELOPMENT OF A SIMPLIFIED STRUCTURAL \\ MODEL)
}

L. Villegas, Prof. Dr. Ing. de caminos

Universidad de Cantabria-España

A. Lijdens, Ing. civil

Fecha de recepción: 28 - I - 94

Universidad Nac. de Cuyo-Argentina

Departamento de Diseno y Construcción de Estructuras de Edificación y Obras Públicas.

E.T.S. de Ingenieros de Caminos, C. y P. de Santander-Universidad de Cantabria

\section{RESUMEN}

El presente trabajo está enmarcado en un programa de investigación desarrollado en el Departamento de Diseño y Construcción de Estructuras de Edificación y Obras Píblicas de la Universidad de Cantabria, sobre las propiedades y posible utilización de estructuras de fábrica en España, y consiste en una comparación de resultados obtenidos con la normativa española (NBE-FL-90 y PIET-70), la europea (Eurocódigo EC6) y la británica (BS5628).

En el artículo se presenta el cálculo de muros portantes de un modelo estructural simplificado realizado con las tres normas analizadas, y mediante la comparación del número máximo de pisos que es posible alcanzar en idénticas condiciones segín cada una de ellas, se demuestra que existen notables diferencias en el resultado dependiendo de la norma con la que se calcule. Se analiza también la relación entre la altura máxima de los muros de arriostramiento del modelo estructural adoptado, su longitud, $y$ la magnitud de la acción horizontal aplicada.

\section{SUMMARY}

This article presents results obtained form a research program, about the properties and possible uses of masonry structures in Spain, developed in the Structural Design and Construction Department of Cantabria University, and consists of a comparison of results obtained using the Spanish (NBE-FL-90 and PIET-70), European (Eurocode EC6) and British (BS5628) standards.

In the article the analysis (with the three considered standards) of load bearing walls in a simplified structural model is presented. Comparing the maximum number of levels that can be achieved with each standard (under the same conditions), it shows that remarkable differences exist in the results. The relationship between the maximum height of the shear walls, their lenght and the magnitude of the applied horizontal load, is also analyzed.

\section{1.-INTRODUCCIÓN}

Si bien la utilización de las estructuras de fábrica en España no está aún muy extendida, éstas presentan múltiples ventajas en el caso de edificios de plantas compartimentadas con luces moderadas y paredes continuas en altura, en las cuales los muros cumplen simultáneamente las funciones de cierre, división del espacio, aislamiento etc. además de soportar las cargas.

(*) Este artículo está basado en el capítulo 4 de la Tesina de Magister realizada por la Ing. Lijdens y dirigida por el Prof. Villegas [4], leída el 15 de Octubre de 1993 en la E.T.S.I.C.C. y P. de Santander, obteniendo la calificación de aptá por unanimidad. 
Existen actualmente dos normas españolas generales para el cálculo de estructuras de fábrica, y se dispone también de un Eurocódigo que está en fase de borrador. Con el objeto de estudiar las consecuencias de la selección de la norma para realizar el cálculo, se comparan en este trabajo los resultados obtenidos con cada una de ellas, habiéndose agregado a esta comparación como parámetro de referencia la normativa inglesa, por ser un país pionero en la investigación del tema y donde el uso de este tipo de estructuras está ampliamente difundido. Así, las normas analizadas son:

1) Eurocódigo EC6: Estructuras de Fábrica - Parte 1 (Norma Europea) [2].

2) PIET-70 y NBE-FL-90 (Normativa Española) [3], [6].

3) BS5628 - Parte 1 (Norma Británica) [1].

El estudio se basó en el cálculo, con las tres normas, de muros de un modelo estructural simplificado, tomándose como parámetro de comparación el número de plantas máximo que podía alcanzarse en las mismas condiciones con cada una de ellas. Adicionalmente, se ha estudiado la influencia que ejercen ciertas variables en las capacidades obtenidas, y se analizó el comportamiento de los muros del modelo adoptado frente a acciones horizontales.

2.-CAPACIDAD DE LOS MUROS SEGÚN LAS DIFERENTES NORMAS. INFLUENCIA DE DIVERSOS PARÁMETROS EN SU VALOR

\section{1.-Modelo estructural y materiales adoptados}

Se adoptó como modelo una estructura ideal simplificada compuesta por cinco muros paralelos en los cuales apoyan los forjados (Fig. 1), de la cual se calcularon los muros exterior (A) e interior (B). Todos los muros tienen el mismo espesor, el cual se toma constante en altura. Las luces de vanos se fijaron en $4,5 \mathrm{~m}$, variándose a $3 \mathrm{~m}$ y $6 \mathrm{~m}$ para estudiar su influencia en las capacidades obtenidas. La longitud de la planta en dirección "Y" se adoptó de $8 \mathrm{~m}$, excepto en el estudio de la longitud necesaria de los muros de arriostramiento, en el que este valor fue variable.

Se han considerado dos tipos de fábrica: de ladrillo y de bloques huecos de hormigón. Las características de las unidades en cuanto a espesores y resistencias, así como el tipo de mortero, se han variado con el fin de estudiar su influencia en la capacidad real de muro. La tabla 1 resume las características de todos los materiales utilizados.

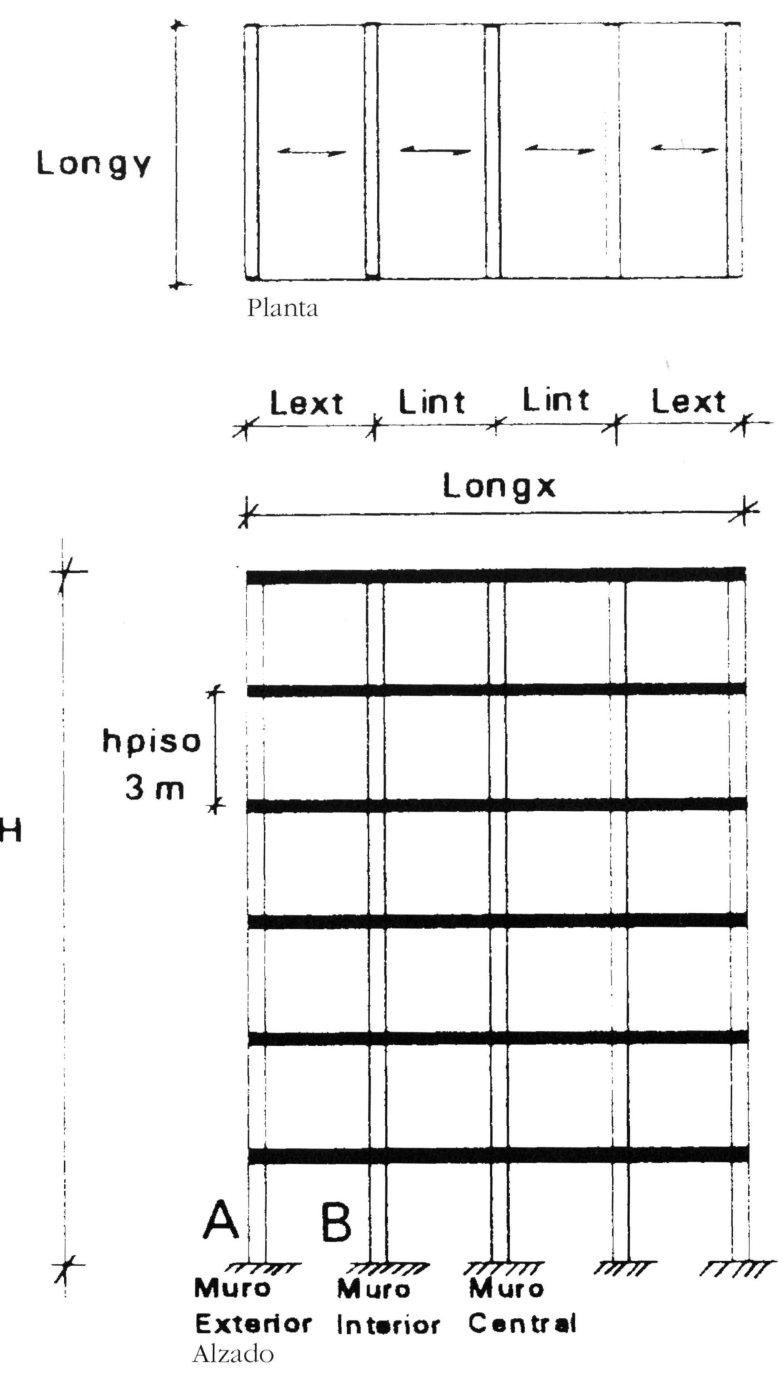

Fig. 1.- Modelo estructural adoptado.

Tabla 1.- Materiales adoptados

\begin{tabular}{|c|c|c||c|}
\hline \multirow{2}{*}{ UNIDAD } & LADRILLO & Espesor $[\mathrm{cm}]$ & $11,5-14$ \\
\cline { 2 - 4 } & & Resistencia $\left[\mathrm{kp} / \mathrm{cm}^{2}\right]$ & $100-300-400-500$ \\
\cline { 2 - 4 } & BLOQUE & Espesor $[\mathrm{cm}]$ & $11,5-14-19-24-29$ \\
\cline { 3 - 4 } $\mathrm{H}^{\circ}$ & Resistencia $\left[\mathrm{kp} / \mathrm{cm}^{2}\right]$ & $60-80-100$ \\
\hline \hline \multicolumn{2}{|c|}{ TIPOS DE MORTERO } & M40-M80-M160 \\
\hline
\end{tabular}

Tabla 2.- Acciones características

\begin{tabular}{|lr|}
\hline Carga permanente & $500 \mathrm{kp} / \mathrm{m}^{2}$ \\
\hline Sobrecarga de uso & $200 \mathrm{kp} / \mathrm{m}^{2}$ \\
\hline Peso especif. muro ladrillo & $1.800 \mathrm{kp} / \mathrm{m}^{3}$ \\
\hline Peso especif. muro bloques & $1.300 \mathrm{kp} / \mathrm{m}^{3}$ \\
\hline Peso revestimiento muros & $50 \mathrm{kp} / \mathrm{m}^{2}$ \\
\hline Zona de viento & Normal \\
\hline Zona de sismo & Grados $6 \mathrm{y} 7$ \\
\hline
\end{tabular}


Tabla 3.- Casos de carga considerados y coeficientes de ponderación de acciones $\mho_{f}$

\begin{tabular}{|c|c|c|c|c|c|}
\hline $\begin{array}{l}\text { CASO de } \\
\text { CARGA }\end{array}$ & $\begin{array}{l}\text { NORMA } \\
\boldsymbol{\gamma}_{f} \text { fg }\end{array}$ & $\begin{array}{c}\text { PERII G } \\
\gamma_{f} \text { fq }\end{array}$ & $\begin{array}{c}\text { DE. ISO Q } \\
\gamma_{f} \text { fw }\end{array}$ & $\begin{array}{l}\text { VIE NTO W } \\
\gamma_{f} \text { fs }\end{array}$ & SISMO S \\
\hline & EC6 & $1,35-1$ & $1,5-0$ & 0 & 0 \\
\hline \multirow[t]{3}{*}{ GQ } & NBE & $1,65-1$ & $1,65-0$ & 0 & 0 \\
\hline & BS5628 & 1,4 & 1,6 & 0 & 0 \\
\hline & EC6 & $1,35-1$ & $1,35-0$ & 1,35 & 0 \\
\hline \multirow[t]{3}{*}{ GQW } & NBE & $1,5-1$ & $1,5-0$ & 1,5 & 0 \\
\hline & BS5628 & 1,2 & 1,2 & 1,2 & 0 \\
\hline & EC6 & $1,35-1$ & 0 & 1,5 & 0 \\
\hline \multirow[t]{2}{*}{ GW } & NBE & $1,5-1$ & 0 & 1,5 & 0 \\
\hline & BS5628 & $1,4-0,9$ & 0 & 1,4 & 0 \\
\hline GQS & EC6-NBE-BSt & 1 & $0,5-0$ & 0 & 1 \\
\hline
\end{tabular}

\section{2.-Acciones consideradas}

Dado que el objeto de este trabajo es comparar resultados obtenidos con diferentes normas en las mismas condiciones con vista a su aplicación en España, la determinación de las cargas características se realizó en todos los casos según normativa española. Las cargas permanentes, y las sobrecargas de uso y de viento se determinaron según NBE-AE88, mientras que las acciones sísmicas se evaluaron con la norma PDS-1-74 partiendo de una intensidad de grado VI o VII (tabla 2).
Las acciones de diseño se determinaron utilizando los coeficientes de mayoración dados por cada código en particular (tabla 3). En el caso de la norma BS5628, se ha comprobado [4] que la combinación de acciones críticas es la de máximo axil, aplicándose por lo tanto los máximos coeficientes de mayoración de acciones verticales (excepto en las verificaciones de tracción).

\section{3.-Determinación de la resistencia de diseño de la fábrica}

La resistencia de diseño de la fábrica, a compresión y a corte, se determinó siguiendo los criterios particulares de cada código, los cuales difieren tanto en lo que respecta a la resistencia característica como a los coeficientes parciales de seguridad.

Si bien la norma española da directamente los valores de diseño, el EC6 y la norma BS5628 establecen la determinación empírica de la resistencia característica, y definen un coeficiente $\boldsymbol{\gamma}_{\mathrm{m}}$ que depende del nivel de control de fabricación y construcción adoptados (excepto BS5628 para el caso de corte, en que establece un coeficiente de 2,5). En los cálculos realizados se asumió un control elevado, que correspondería al tipo "A" del EC6 y al "Especial" de BS5628, por lo que los coeficientes $\gamma_{\mathrm{m}}$ adoptados fueron de 2 para EC6 y 2,5 para BS5628.
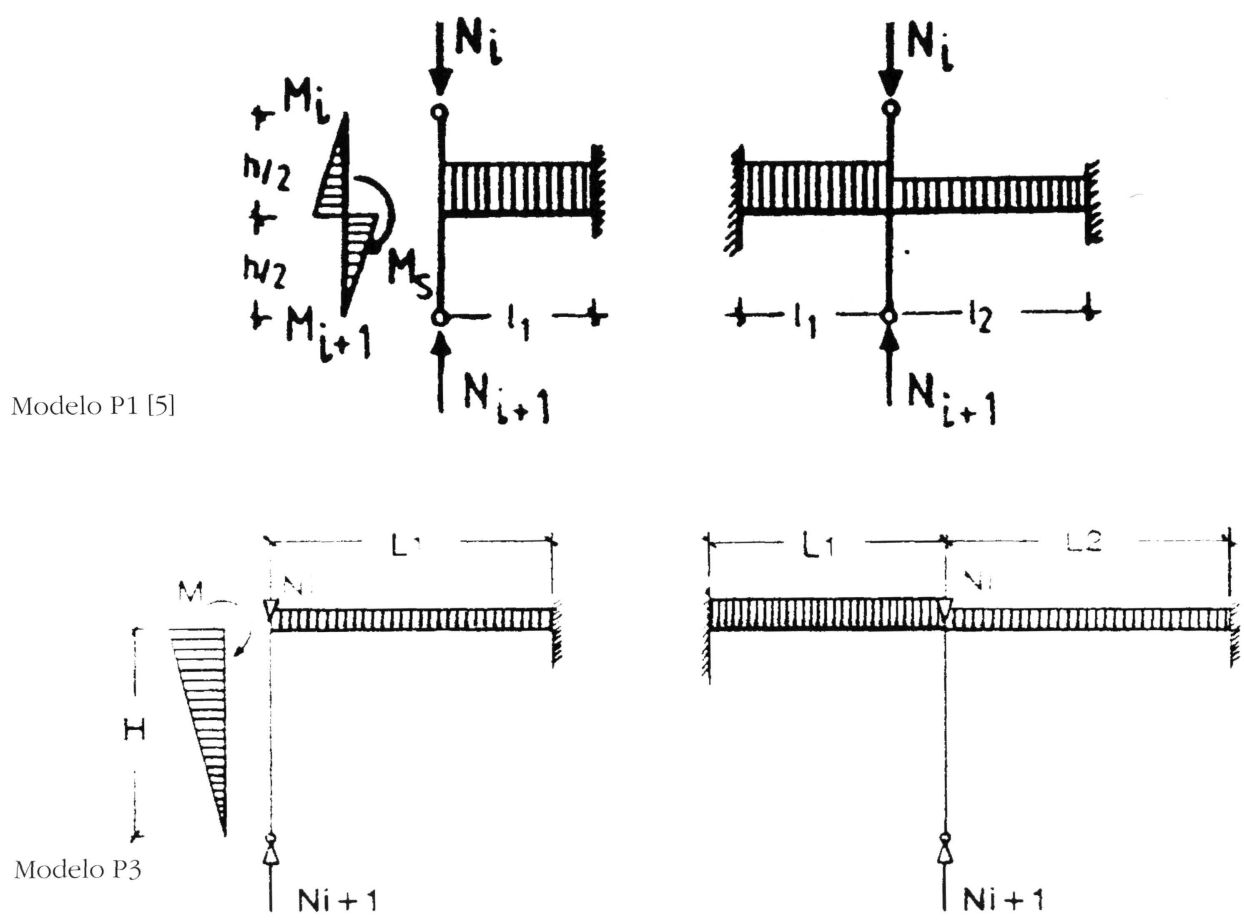

Fig. 2.- Modelos elásticos adoptados para la resolución con EC6. 
Respecto a la norma NBE, en el caso de ladrillos de resistencia mayor que $300 \mathrm{kp} / \mathrm{cm}^{2}$ (valor máximo considerado por la misma en sus tablas), la resistencia a compresión de diseño se obtuvo para mortero M80, espesor de junta de 1 a 1,5 cm y consistencia sograsa del mortero, mediante una ley exponencial ajustada por mínimos cuadrados.

\section{4.-Modelos de cálculo}

En el cálculo de muros portantes existen dos efectos que reducen la capacidad de carga de los mismos: la excentricidad con que actúa la carga vertical, y la esbeltez del muro que aumenta la posibilidad del pandeo. Para la evaluación de la excentricidad de la carga se han seguido los criterios dados por cada norma.

El EC6 establece un método simplificado que consiste en considerar que la carga del forjado está aplicada al 5\% de la luz del vano en muros exteriores, o el 5\% de la diferencia de luces en muros que reciben forjados de ambos lados. Paralelamente admite la posibilidad de calcular las excentricidades mediante una distribución elástica, por lo que en los cálculos realizados se han adoptado, además del modelo simplificado ya descrito, los dos modelos elásticos de la figura 2, el primero de ellos (P1) propuesto por Mann [5], en el que se suponen todos los nudos rígidos, y el segundo (P3) en el que se asume que la carga actúa centradamente en la sección inferior del muro.

La normativa española NBE (PIET-70 y FL-90) define un modelo simplificado de distribución elástica según las rigideces relativas de forjado y muro: mientras que la norma BS5628 considera que la carga del forjado está aplicada a una distancia de un tercio de la zona de apoyo del mismo, medida desde el borde cargado del muro.

Mediante la elaboración de un programa de ordenador, se determinó la cantidad de plantas máxima a soportar por los muros exterior (más solicitado por excentricidad) e interior (con máximo axil), realizándose piso a piso todas las verificaciones en estado límite último por agotamiento resistente de la sección (superior, media e inferior), contempladas por cada normativa en particular.

\section{5.-Resultados}

Las figuras 3 y 4 muestran la variación del número máximo de plantas alcanzado por los muros exte-
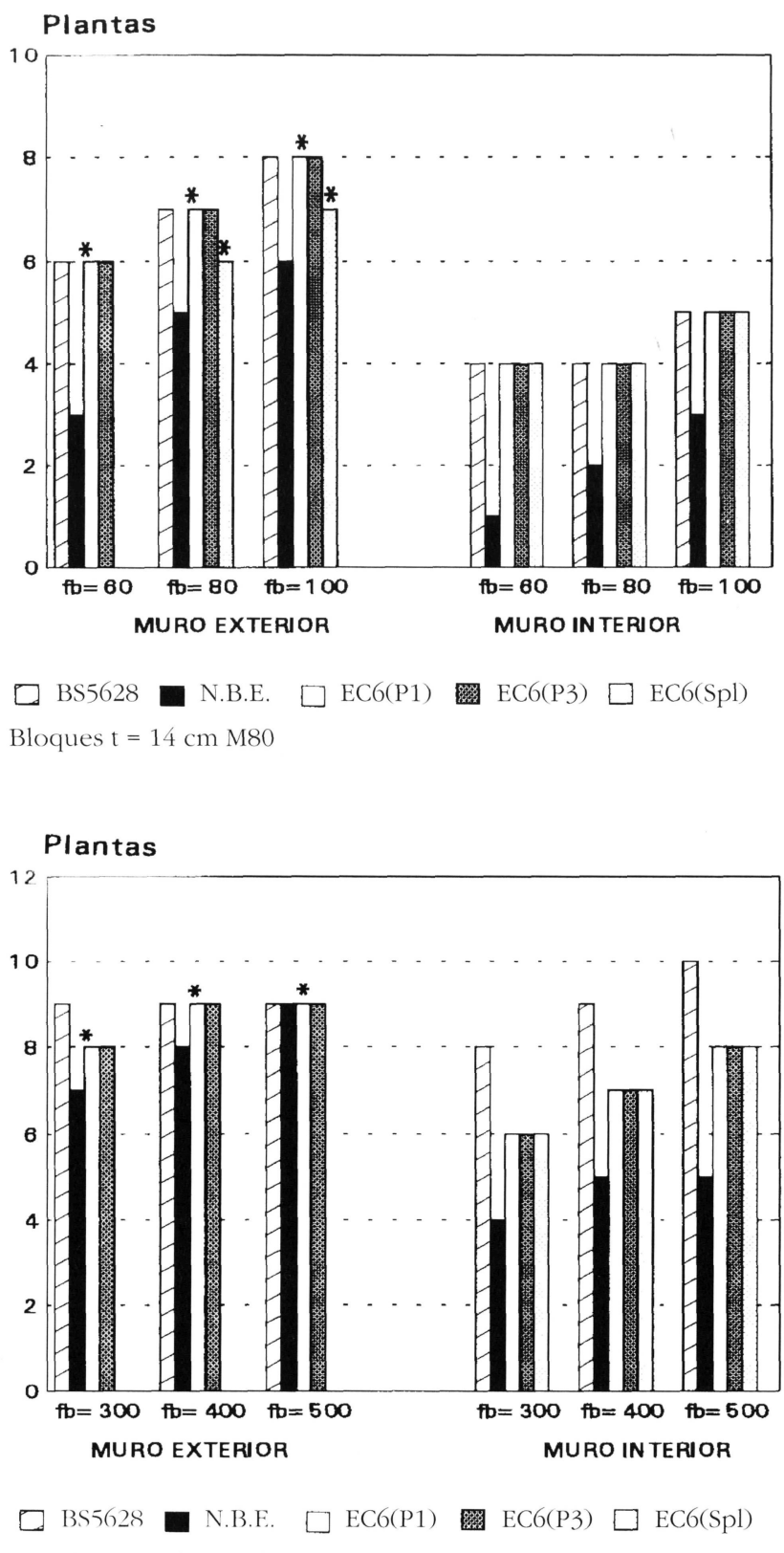

Ladrillos $\mathrm{t}=1+\mathrm{cm} \mathrm{M80}$

Fig. 3.- Variación de las alturas alcanzadas por los muros con la resistencia de la unidad "fb".

rior e interior con la resistencia a compresión y con el espesor de la unidad, para la estructura tipo de 4,5 $\mathrm{m}$ de luz de vanos.

En los casos señalados con un asterisco, que corresponden todos al EC6 en el muro exterior, las capacidades obtenidas con la aplicación exacta de la norma fueron nulas. Esto es debido a las grandes excentricidades dadas por los diferentes modelos adoptados con el EC6, muchemayores que las obtenidas con las normas NBE y BS5628, las cuales son muy similares entre sí [4]. 
En estos casos se realizó un cálculo alternativo agregando como única variante la limitación de la excentricidad máxima al 45\% del espesor del muro "t", valor que se consideró imposible de ser superado debido a las fisuraciones y reacomodaciones que se producirían en el material. Los resultados así obtenidos se señalan con un asterisco para indicar que en estos casos la capacidad obtenida según indica dicha norma sería nula.

* Puede observarse (Figs. 3 y 4) que salvo los casos mencionados anteriormente, las alturas obtenidas con los diferentes modelos del EC6 y con BS5628 son bastante similares. Como contrapartida, la norma $N B E$ ha dado capacidades del orden de 2 plantas menos que el resto, presentando por lo tanto una tendencia mucho más conservadora, sobre todo para los muros de menor espesor (más esbeltos).

* Un incremento de la resistencia a compresión (Fig. 3) o del espesor de la unidad (Fig. 4) conduce a significativos aumentos en el número de plantas que es posible alcanzar.

* Las capacidades alcanzadas por el muro interior son menores que las del muro exterior, salvo en el caso de alturas importantes [4], donde la magnitud de la acción horizontal limita por tracción el número de plantas máximo para el muro exterior, poco cargado. En estos casos, no es posible aumentar la altura del muro mediante una mejora en la calidad de los materiales.

* Como se muestra en la figura 5, la incidencia de la calidad del mortero en la capacidad del muro es moderada.

* Un aumento de $3 \mathrm{~m}$ a $6 \mathrm{~m}$ en la luz del vano produce disminuciones importantes de la capacidad, del orden de 2 a 3 plantas (Fig. 6).

* A efectos comparativos y con un interés puramente teórico, se ha calculado la capacidad máxima obtenida para los muros exterior e interior en ausencia de acciones horizontales, con fábrica de ladrillo de $14 \mathrm{~cm}$ de espesor y 300 $\mathrm{kp} / \mathrm{cm}^{2}$ de resistencia, y mortero M80. La tabla 4 muestra los resultados obtenidos. En el caso del Eurocódigo los resultados mostrados corresponden al modelo P3. Puede verse que mientras las alturas dadas por EC6 y BS5628 son bastante similares, las capacidades máximas obtenidas con la norma española son del orden del 50\% de las anteriores.

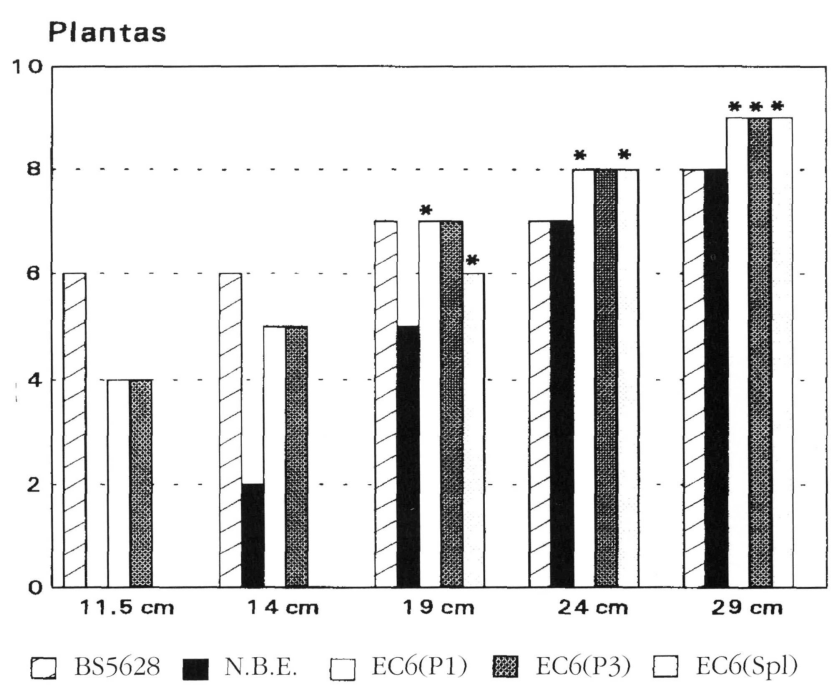

Bloque $\mathrm{fb}=80 \mathrm{kp} / \mathrm{cm}^{2} \mathrm{M} 80$ muro exterior

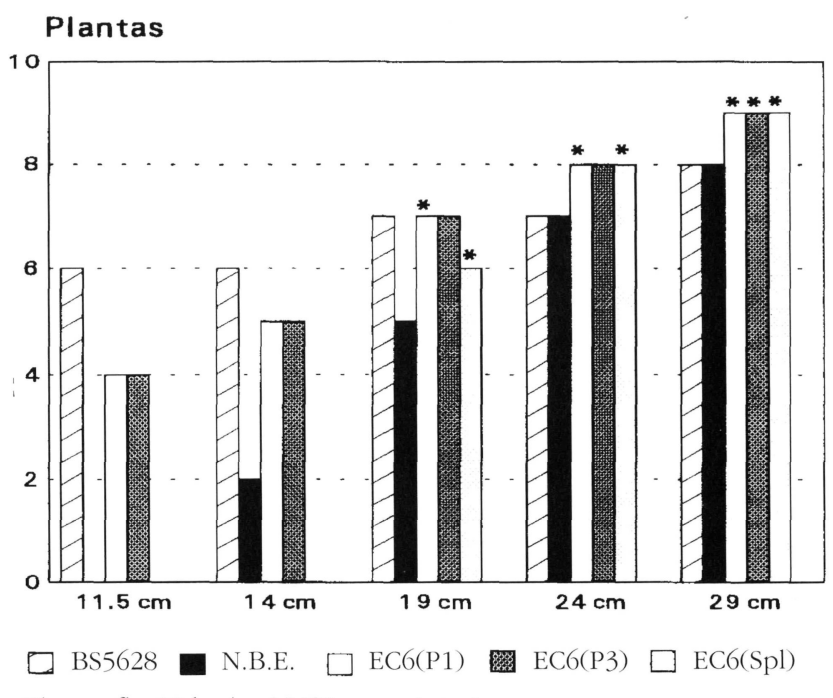

Bloque $\mathrm{fb}=80 \mathrm{kp} / \mathrm{cm}^{2} \mathrm{M} 80$ muro interior

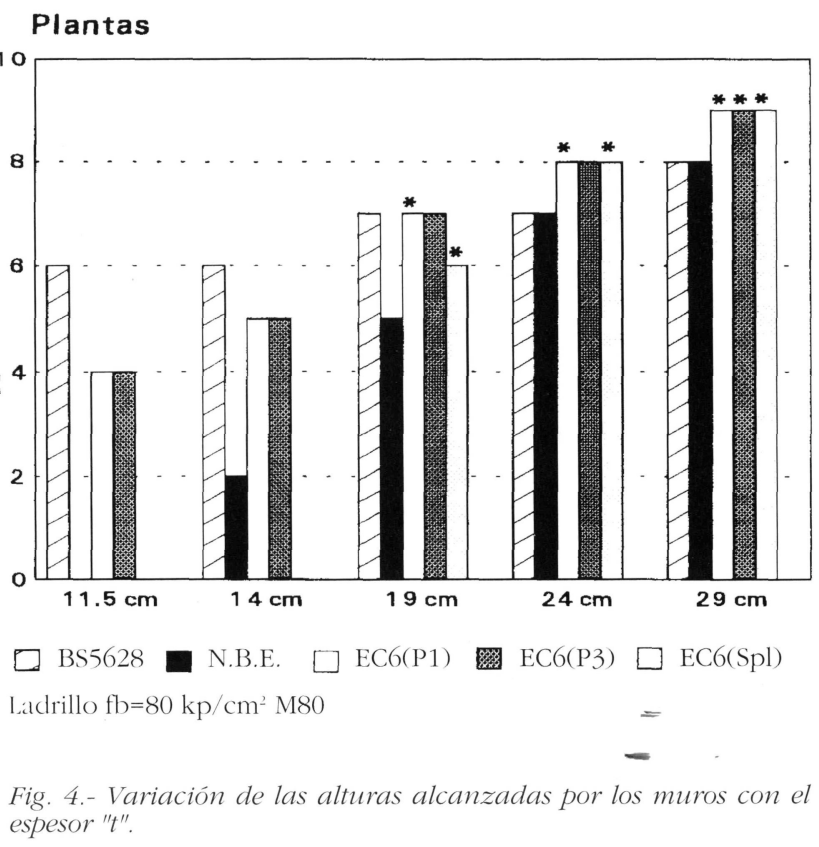



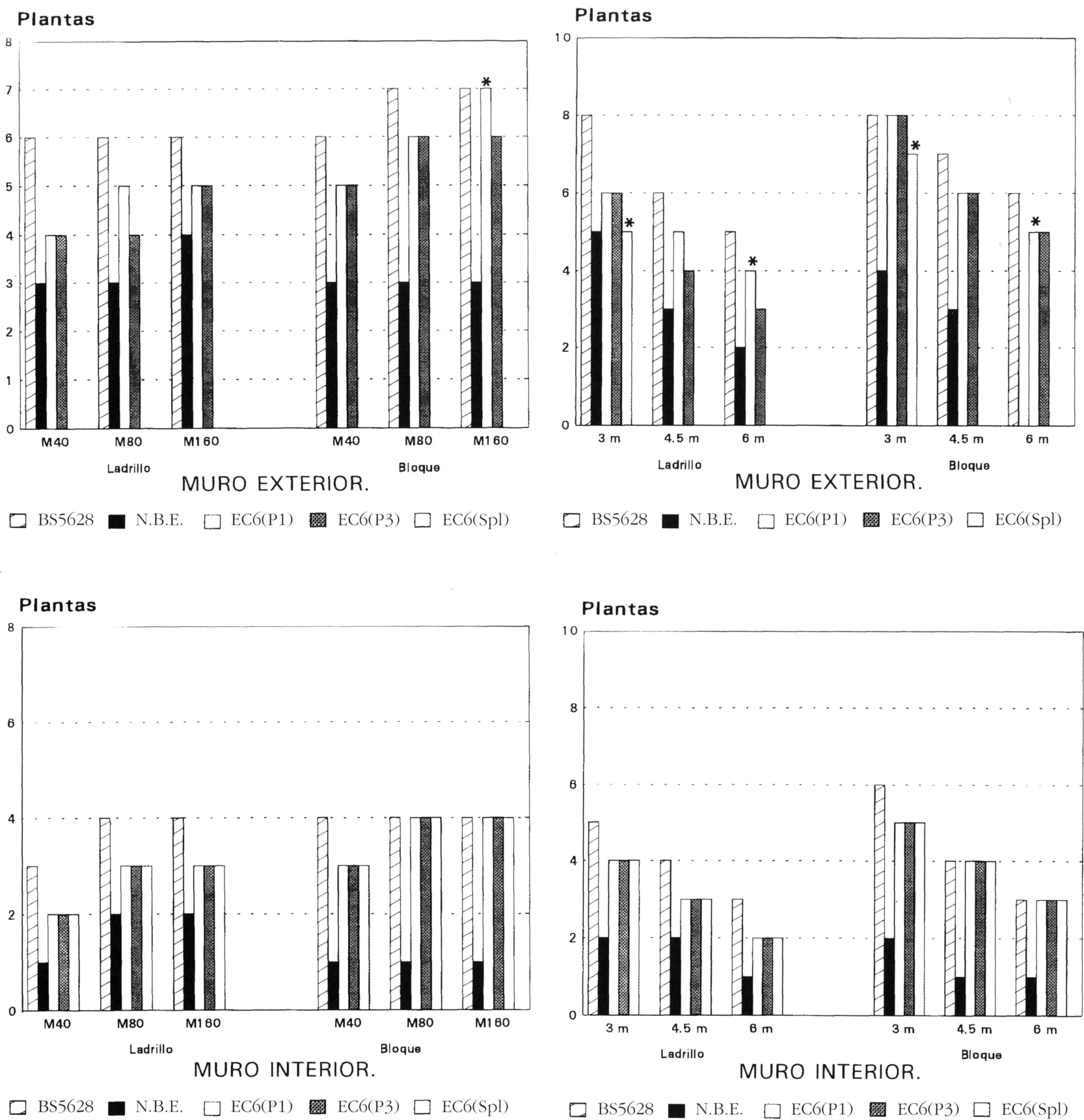

Fig. 5.- Variación de las alturas alcanzadas con el tipo de mortero $\left(\mathrm{fb}=100 \mathrm{kp} / \mathrm{cm}^{2}-\right.$ Espesor $\left.=14 \mathrm{~cm}\right)$.

Fig. 6.- Variación de las alturas alcanzadas con la luz del vano $\left(\mathrm{fb}=100 \mathrm{kp} / \mathrm{cm}^{2}-\right.$ M8O - Espesor $\left.=14 \mathrm{~cm}\right)$.

Tabla 4.- Capacidad máxima de los muros a cargas verticales

- Ladrillo $\mathrm{f}_{\mathrm{b}}=300 \mathrm{kp} / \mathrm{cm}^{2}-\mathrm{t}=14 \mathrm{~cm}$-Mortero M80

\begin{tabular}{|c||c|c|}
\hline \multirow{2}{*}{ NORMA } & MURO EXTERIOR & MURO INTERIOR \\
\hline \hline BS5628 & 15 & 8 \\
\hline NBE & 7 & 4 \\
\hline EC6 & 13 & 7 \\
\hline
\end{tabular}

\section{6.-Verificaciones críticas más frecuentes}

En todos los cálculos se ha realizado un control de las causas que limitaron la capacidad portante de los muros. La tabla 5 muestra a modo de ejemplo, para fábrica de bloques de $19 \mathrm{~cm}$ de espesor, las alturas máximas alcanzadas con diferentes resistencias del bloque $\mathrm{f}_{\mathrm{b}}$, detallándose las verificaciones que no cumplieron al darle al muro una planta más de la altura indicada. Puede observarse que en la 
Tabla 5.- Alturas máximas alcanzadas - Fábrica de bloques - $($ M80-t=19cm $)$

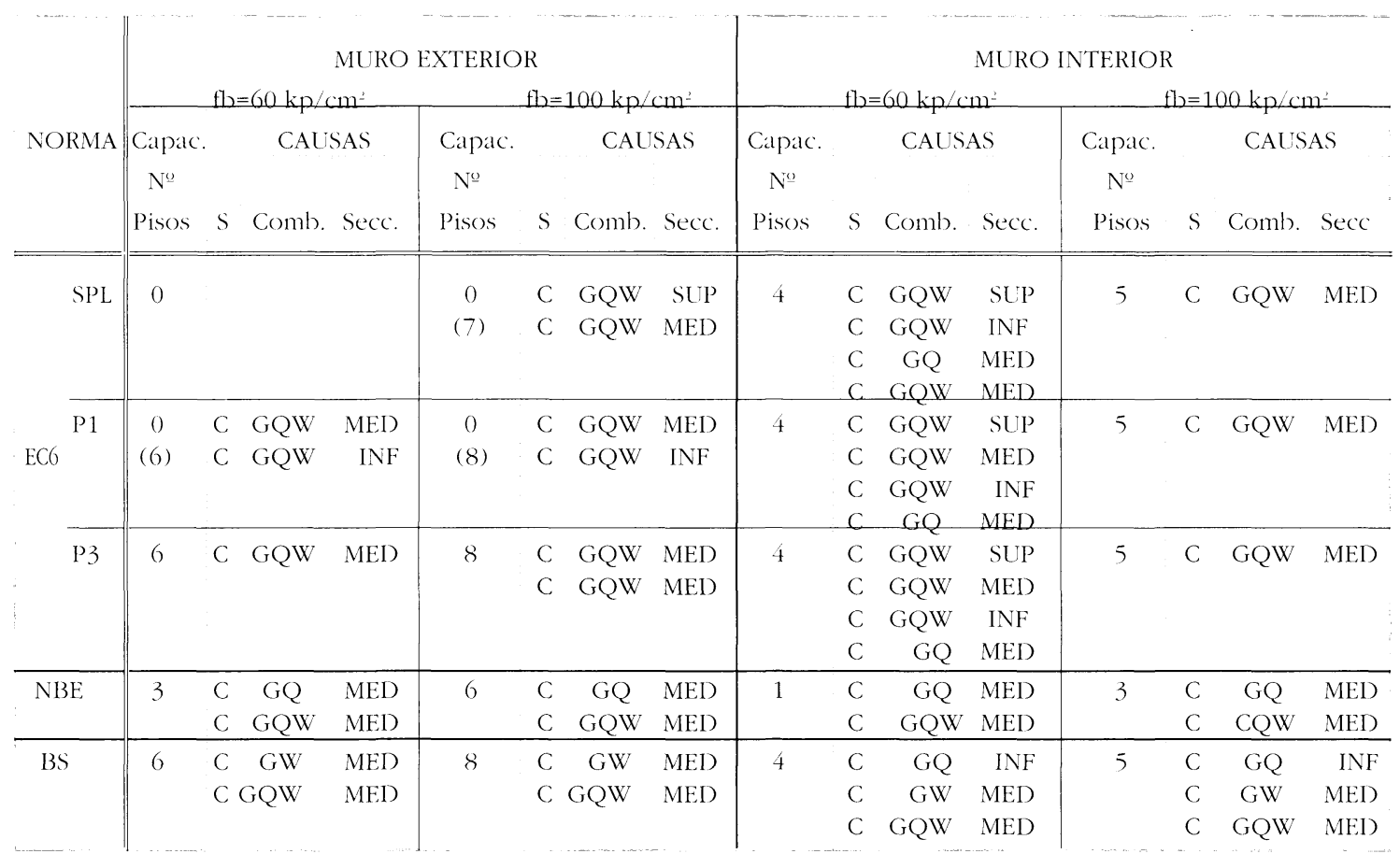

mayoría de las ocasiones se producía simultáneamente más de una falla.

Dicho detalle se realizó en 3 subcolumnas, de la siguiente manera:

1) Se indica con "S" a la solicitación que no verificó, la cual ha sido Compresión (C) o Tracción (T) en todos los casos.

2) Se indica con "Comb" a la combinación de acciones para la cual se produjo la falla, correspondiendo "G" a cargas permanentes, "Q" a sobrecarga de uso, "W" a viento y "S" a sismo.

3) "Secc" indica la sección de muro donde se produjo la falla (SUP, MED e INF).

Los valores indicados entre paréntesis en la tabla corresponden a los cálculos realizados para EC6 en el muro exterior, en los que se consideró una limitación del valor máximo de la excentricidad al 45\% del espesor (apartado 2.5)

Se ha obtenido como combinación de acciones crítica más frecuente la combinación "Carga Permanente+Sobrecarga+Viento" $(G+Q+W)$. La sección que más frecuentemente resultó determinante ha sido la sección media. Respecto a los esfuerzos, no se han registrado fallas por corte, y las fallas por tracción han comenzado hacia una altura de 8 ó 9 plantas en el muro exterior, menos cargado.

\section{3.-ESBELTEZ COPLANAR DE LOS MUROS DE ARRIOSTRAMIENTO}

\section{1.-Esbeltez coplanar de los muros}

Con objeto de encontrar la relación que existe entre la longitud de los muros del modelo utilizado y su capacidad para resistir cargas horizontales, así como de estudiar las causas de falla en caso de que el muro no tenga la longitud suficiente, se ha calculado para cada altura del mismo la longitud mínima que se requiere para cumplir con todas las verificaciones de seguridad.

Se determinó así la esbeltez coplanar máxima con la que los muros del modelo pueden resistir las acciones horizontales, llamando "esbeltez coplanar" al cociente entre la altura del muro, y su longitud (H/longy, figura 1).

\section{2.-Modelo y materiales adoptados}

El modelo estructural adoptado es el de la figura 1, con una luz de todos los vanos de $4,5 \mathrm{~m}$. La longitud en dirección "Y" del modelo se varió para encontrar la mínima necesaria para cada altura. Para el Eurocódigo EC6, se adoptó en este desarrollo el modelo P3, ya que ha sido el que dio los resultados más acordes con las otras normas, de los tres modelos utilizados anteriormente. 
Tabla 6.- Longitud mínima necesaria de los muros de arriostramiento para cada número $\mathbf{N}$ de plantas

\begin{tabular}{|c|c|c|c|c|c|c|c|}
\hline \multirow[t]{2}{*}{$\begin{array}{l}\text { TIPO de } \\
\text { MURO }\end{array}$} & \multirow[t]{2}{*}{$\mathrm{N}$} & \multicolumn{3}{|c|}{$\begin{array}{l}\text { VIENTO ZONA } \\
\text { NORMAL }\end{array}$} & \multicolumn{3}{|c|}{$\begin{array}{c}\text { VIENTO ZONA } \\
\text { EXPUESTA }\end{array}$} \\
\hline & & BSt & NBE & EC6 & BSt & NBE & EC6 \\
\hline \multirow[t]{14}{*}{ EXTERIOR } & 2 & 3 & 3,5 & 3 & 4,5 & 5 & 4,5 \\
\hline & 3 & 4 & 4 & 4 & 5,5 & 5,5 & 5,5 \\
\hline & 4 & 5,5 & 5,5 & 5,5 & 6 & 6 & 6 \\
\hline & 5 & 6 & 6 & 6 & 7 & 7 & 7 \\
\hline & 6 & 6,5 & 6,5 & 6,5 & 7,5 & 7,5 & 7,5 \\
\hline & 7 & 7 & 8 & 7 & 8 & 9 & 8 \\
\hline & 8 & 7,5 & - & 7,5 & 8,5 & - & 8,5 \\
\hline & 9 & 8 & - & 8,5 & 9 & - & 9,5 \\
\hline & 10 & 8,5 & - & 10,5 & 9,5 & - & 12 \\
\hline & 11 & 10 & - & 15 & 11,5 & - & 17 \\
\hline & 12 & 11 & - & 20,5 & 12,5 & - & 23 \\
\hline & 13 & 13 & - & 35 & 14,5 & - & 37,5 \\
\hline & 14 & 15,5 & - & - & 17,5 & - & - \\
\hline & 15 & 19,5 & - & - & 21,5 & - & - \\
\hline \multirow[t]{7}{*}{ INTERIOR } & 2 & 2 & 2,5 & 2,5 & 3,5 & 3,5 & 3,5 \\
\hline & 3 & 3 & 3 & 3 & 4 & 4 & 4 \\
\hline & 4 & 4 & 5 & 4 & 4,5 & 6 & 4,5 \\
\hline & 5 & 4,5 & - & 5 & 5 & - & 5,5 \\
\hline & 6 & 5 & - & 7,5 & 5,5 & - & 8,5 \\
\hline & 7 & 6 & - & 14,5 & 7 & - & 17 \\
\hline & 8 & 8 & - & - & 9 & - & - \\
\hline
\end{tabular}

Respecto a los materiales, y dado que el interés de este análisis es puramente teórico, se ha realizado únicamente para el caso particular de fábrica de ladrillo de $300 \mathrm{kp} / \mathrm{cm}^{2}$ de resistencia, mortero M80 y espesor de $14 \mathrm{~cm}$.

\section{3.-Resultados}

Para los muros exterior e interior del modelo analizado se calculó, para cada número de plantas, la longitud mínima con que el muro cumple todas las verificaciones, determinándose así la esbeltez coplanar necesaria para resistir las acciones horizontales.

Para ello, partiendo de una altura de dos niveles, para cada número de plantas se fue aumentando la longitud de los muros en incrementos de a $0,5 \mathrm{~m}$, hasta lograr que el muro resistiera esa altura. Se procedió así hasta que el muro fallara por cargas verticales únicamente, llegándose a la máxima capacidad admisible por el mismo para el caso estudiado.

La tabla 6 muestra la longitud mínima de muro necesaria para que los muros de arriostramiento soporten el número de plantas indicado. En las figuras 7 y 8 se muestra además cuál es la causa que provocaría la falla en caso de que el muro no tuviera la longitud suficiente para esa altura.

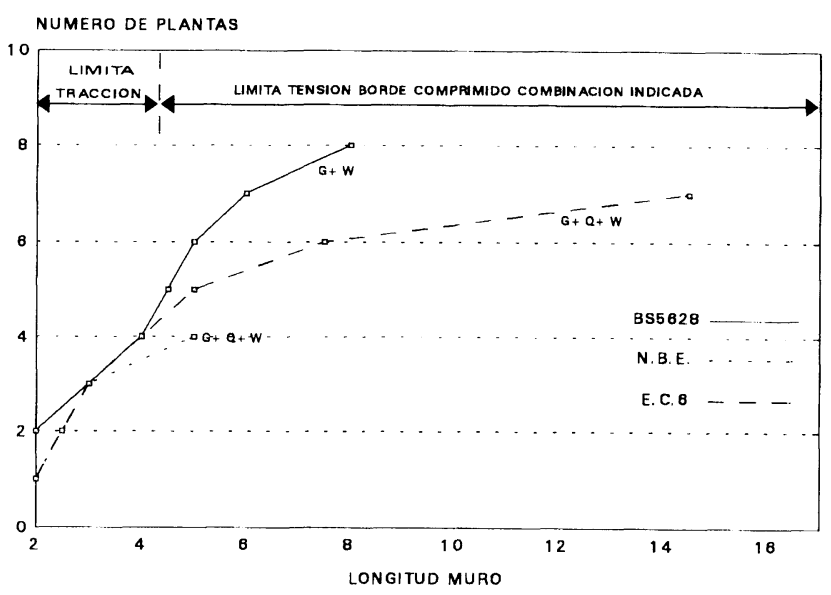

ZONA VIENTO NORMAL

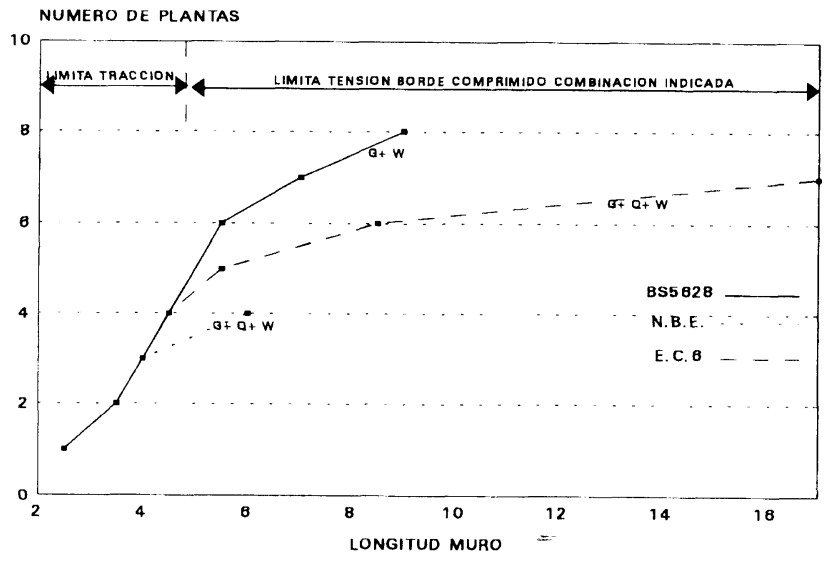

ZONA VIENTO EXPUESTA

Fig. 7.- Longitud mínima de los muros de arriostramiento para cada altura. Muro interior. 


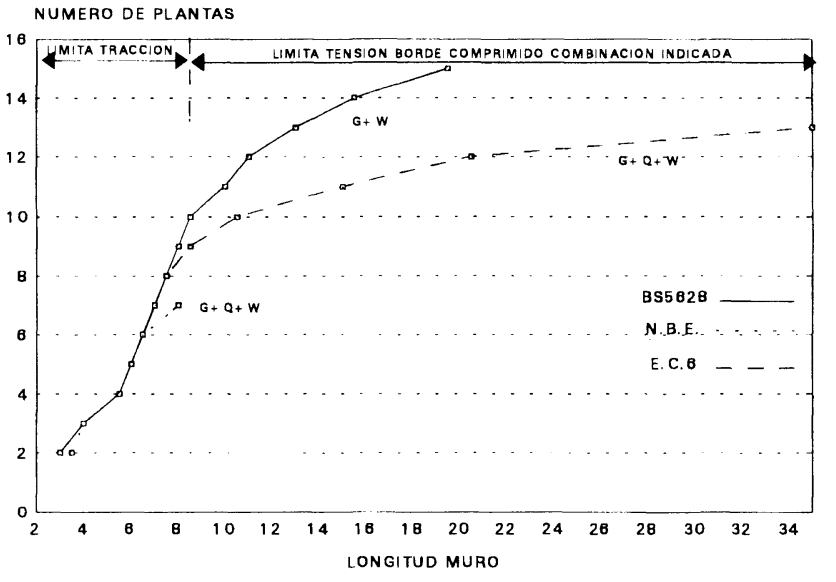

ZONA VIENTO EXPUESTA

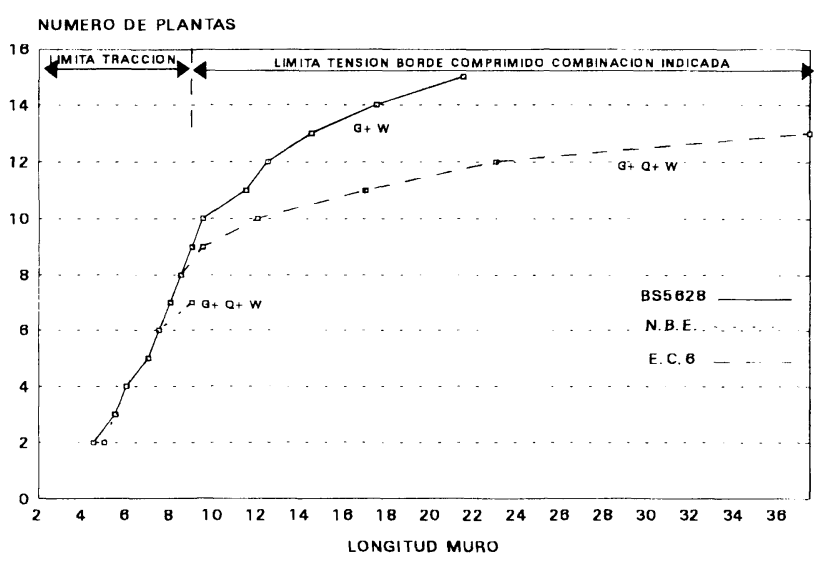

ZONA VIENTO EXPUESTA

Fig. 8.- Longitud mínima de los muros de arriostramiento para cada altura. Muro exterior.

\section{4.-CONCLUSIONES}

1) La influencia de la norma de cálculo en la capacidad de carga de los muros de fábrica simple es importante. En ciertos casos de muros exteriores, el cálculo con el Eurocódigo conduce a capacidades nulas contrariamente al resto de las normas (aún las más conservadoras), obteniéndose resultados más similares al resto mediante la limitación del valor máximo de la excentricidad de la carga.

La normativa española NBE-FL-90 y PIET-70 presenta una tendencia marcadamente conservadora sobre todo en el caso de muros esbeltos, dando capacidades a cargas verticales del orden del $50 \%$ de las dadas por EC6 y BS5628, las cuales en general presentaron resultados muy similares.

2) De acuerdo a los resultados obtenidos, se han alcanzado los siguientes números de plantas:

* En fábrica de bloques de resistencias usuales, de 1 a 3 plantas con espesor de $14 \mathrm{~cm}$ hasta 5 a 6 plantas con $29 \mathrm{~cm}$ de espesor, para el muro interior; y de 2 a 6 plantas con $14 \mathrm{~cm}$ hasta 8 a 9 plantas con $29 \mathrm{~cm}$ en el muro exterior, dependiendo de la norma de cálculo.

* En fábrica de ladrillos de calidad corriente, de 2 a 6 plantas con $11,5 \mathrm{~cm}$ de espesor y 4 a 8 plantas con $14 \mathrm{~cm}$ en el muro interior; y de hasta 8 plantas con $11,5 \mathrm{~cm}$ y 9 plantas con $14 \mathrm{~cm}$ de espesor en el muro exterior, según la norma de cálculo.
3) Un incremento de la resistencia a compresión o del espesor de la unidad aumenta significativamente la capacidad del muro. La influencia en la misma de la calidad del mortero utilizado es mucho más moderada.

4) El número de plantas máximo alcanzado por los muros varía sensiblemente con la luz de los vanos soportados por el mismo, habiéndose registrado disminuciones del orden de 2 a 3 plantas al aumentar la misma de $3 \mathrm{~m}$ a $6 \mathrm{~m}$.

5) Para alturas del orden de hasta 4 plantas en el muro interior, y 8 plantas en el exterior, las esbelteces coplanares límite de los muros del modelo coinciden bastante con las diferentes normas.

6) En zona de viento normal, para alturas bajas y medias, la longitud necesaria de los muros de arriostramiento del modelo utilizado es del orden de $1 \mathrm{~m}$ por cada planta de altura del mismo. En el caso de zona de viento expuesta las longitudes requeridas son algo mayores.

\section{5.-AGRADECIMIENTOS}

Los autores quieren expresar su agradecimiento al Departamento de Diseño y Construcción de Estructuras de Edificación y Obras Públicas de la Universidad de Cantabria, donde se ha llevado a cabo este trabajo, como parte de un programa de 
investigación sobre las estructuras de fábrica, y a la Universidad de Cantabria y la empresa TECONSA (Tecnología de la Construcción S.A.) quienes han financiado los estudios realizados.

\section{6.-BIBLIOGRAFIA}

[1] BRITISH STANDARD INSTITUTION; "Code of practice for the use of masonry-BS5628 (Part 1)"Londres, 1985.

[2] COMMISSION OF EUROPEAN COMMUNITIES; "Eurocode EC6 -Common unified rules for unreinforced masonry structures- Part 1"- Bruselas, 1988.

[3] Instituto EDUARDO TORROJA; "PIET 7O, Obras de fábrica" - Prescripciones del Instituto Eduardo Torroja, Madrid, 1971.
[4] LIJDENS. A.; "Estudio de las posibilidades de utilización de estructuras de fábrica simple en edificios de altura según diferentes normas" - Tesina de Magister (Director: Villegas, L) - Departamento de Diseño y Construcción de Estructuras de Edificación y Obras Públicas, Universidad de Cantabria, Santander, 1993.

[5] MANN. W.; "Basics of design of masonry walls subjected to vertical loads and buckling according to the present draft of Eurocode EC6 and comparison with experimental data". Proceedings of the 9th International Brick/Block Masonry Conference. Berlín, 1991.

[6] MINISTERIO DE OBRAS PUBLICAS Y URBANISMO; "NBE-FL-90, Muros resistentes de fábrica de ladrillo". B.O.E. 1990.01.04, Madrid, 1990.

[7] VILLEGAS. L.; "Estructuras de fábrica" - Servicio de Publicaciones, E.T.S. de Ing. de Caminos, Canales y Puertos, Universidad de Cantabria, 1992.

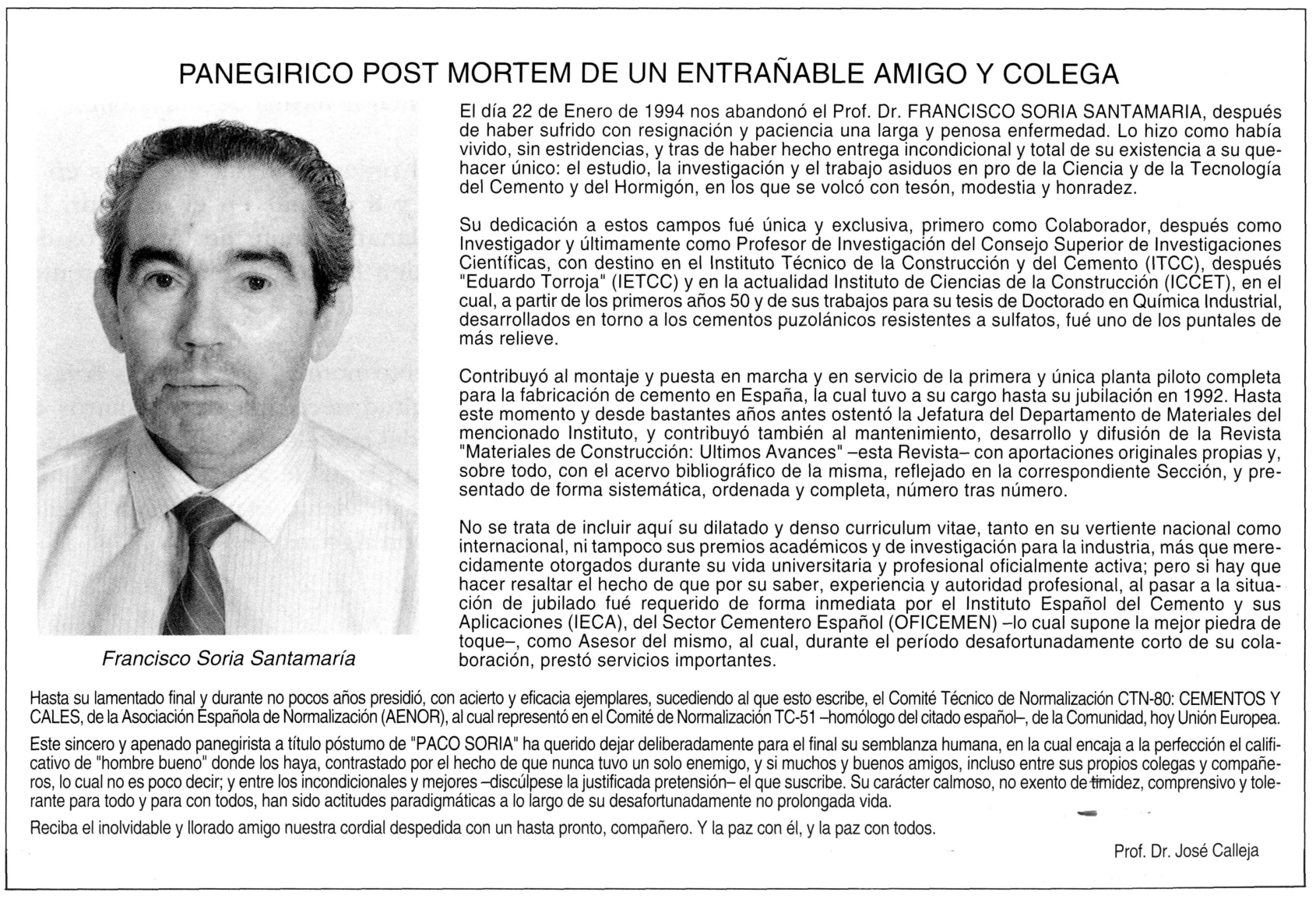

